\title{
Alteración de mediciones radiográficas en osteosíntesis de húmero proximal con clavo centromedular
}

\author{
Alteration of X-ray measurements in proximal humerus osteosynthesis with intramedullary nail
}

\author{
Valencia-Ramón EA, * Hazan-Lasri E, ${ }^{\ddagger}$ Pineda-Gómez E, ${ }^{\S}$ García-Martínez E, Ponce-De León Domínguez J, \\ Gutiérrez-Sevilla G, Gutiérrez-Báez J, ${ }^{\|}$Moreno-Degante L, Ruiz-Suárez M ${ }^{\|}$
}

Instituto Nacional de Rehabilitación.

RESUMEN. Introducción: La calidad de reducción en las fracturas de húmero proximal es posible valorarla a través de criterios radiográficos $(\mathrm{ACD}=$ ángulo cervicodiafisario; $\mathrm{OM}=$ offset medial; DTC = distancia tuberosidadcabeza y ES = espacio subacromial), los cuales predicen la funcionalidad. Sin embargo, estos criterios establecidos para otros implantes no están descritos para el clavo centromedular. Material y métodos: Estudio de cohorte observacional y retrospectivo en pacientes $>18$ años con osteosíntesis con clavo centromedular (durante el período de 2014 a 2017), en donde se evaluaron las características demográficas, severidad de lesión (clasificación de Neer) y las mediciones radiográficas progresivas y complicaciones. Resultados: Se analizaron 84 casos, cuya edad en promedio fue de $65 \pm 2$ años y la media de seguimiento de 13.9 meses. Por clasificación de Neer: $40.4 \%$ fueron grado II; $29.7 \%$, grado III y $29.7 \%$, grado IV. La consolidación en varo aumenta $10.7 \%$ al final del seguimiento, siendo aquellos pacientes Neer grado III y IV los más frecuentes. La deformidad $>140^{\circ}$ predomina para Neer grado II $(n=15 / 34)$. En $30.9 \%$, se presenta un cambio de ACD $(\triangle \mathrm{ACD}) \geq 10^{\circ}$. En general, $86.9 \%$ tiene cambios de DTC $<5 \mathrm{~mm}$. En los casos Neer grado III/IV, 32\% tiene una alteración de ES $>$ $5 \mathrm{~mm}$. La complicación más frecuentemente es la necrosis avascular (NAV) con $44 \%$ de los casos. Conclusiones: Las
ABSTRACT. Introduction: The quality of reduction in proximal humerus fractures is valuable with radiographic criteria (Neck-shaft angle [NSA]; medial offset [MO]; distance head tuberosity [DHT] and subacromial space [SS]) that predict functionality. These criteria set for other implants are not described for the intramedullary nail. Material and methods: Observational cohort study, retrospective, with patients $>18$ years, with osteosynthesis with intramedullary nail (2014 to 2017), evaluating demographic characteristics, severity of injury (Neer classification), progressive radiographic measurements and complications. Results: 84 cases with an average age of 65 \pm 2 years and an average follow-up of 13.9 months were analyzed. By Neer rating $40.4 \%$ were grade II, $29.7 \%$ grade III and $29.7 \%$ grade IV. Consolidation in varus increases to $10.7 \%$ at the end of follow-up, with Neer III and IV patients. The $>140^{\circ}$ deformity is prevalent for Neer II $(\mathrm{n}=$ $15 / 34$ ). $30.9 \%$ have a change of neck-shaft angle of $\geq 10^{\circ}$. Overall $86.9 \%$ has HTD changes $<5 \mathrm{~mm}$. Cases Neer III/ IV $32 \%$ has alteration of SS $>5 \mathrm{~mm}$. The most common complication is avascular necrosis (AVN) with $44 \%$ of cases. Conclusions: Radiographic measurements have changes in long-term follow-up. Alterations in NSA $\left(>10^{\circ}\right)$ and SS ( $>5 \mathrm{~mm}$ ) occur in higher proportion of Neer III/IV patients, according to the severity of the fracture, favoring

\section{Nivel de evidencia: IV}

* Residente de Ortopedia.

* Jefe de División de Traumatología, Urgencias e Infecciones Óseas.

$\S$ Jefe de Servicio de Traumatología.

" Médico adscrito al Servicio de Traumatología.

Dirección para correspondencia: Dr. Edwin Alfonso Valencia-Ramón Av. México-Xochimilco Núm. 289, Col. Arenal de Guadalupe, C. P. 14389, Alcaldía Tlalpan, CDMX. Teléfono: 599910 00, ext. 12412.

E-mail: edwin.valenc@gmail.com

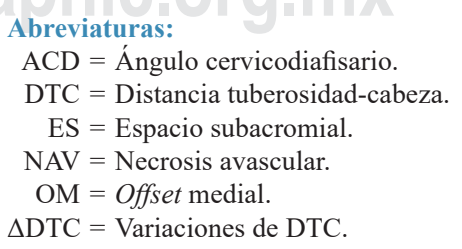

https://dx.doi.org/10.35366/95319 doi: $10.35366 / 95319$ 
mediciones radiográficas presentan cambios en valoraciones a largo plazo. Las alteraciones en $\operatorname{ACD}\left(>10^{\circ}\right)$ y $\operatorname{ES}(>$ $5 \mathrm{~mm}$ ) se dan en mayor proporción en aquellos pacientes Neer grado III/IV (acorde con la severidad de la fractura), lo que favorece consolidaciones viciosas en varo o valgo y una mayor presencia de NAV.

Palabras clave: Fractura del húmero proximal, tratamiento, clavo, complicaciones, radiografías.

\section{Introducción}

Las fracturas de húmero proximal representan de 4 a $5 \%$ del total de todas las fracturas en los adultos, con una mayor prevalencia para el sexo femenino y para la tercera edad. En la actualidad, la osteosíntesis de húmero proximal sigue siendo controversial. Uno de los tratamientos quirúrgicos es el clavo centromedular, el cual ofrece una disminución de tiempo quirúrgico y una menor lesión en los tejidos blandos. ${ }^{1,2}$

En las fracturas de húmero proximal, la calidad de la reducción y las propiedades biomecánicas de la fijación son más importantes que la magnitud de desplazamiento inicial de la fractura y es posible valorarlas a través de criterios radiográficos $(\mathrm{ACD}=$ ángulo cervicodiafisario; $\mathrm{OM}=$ offset medial; DTC = distancia tuberosidad-cabeza y ES = espacio subacromial), los cuales predicen la funcionalidad a largo plazo. ${ }^{3,4}$ Estos criterios ya están establecidos para otros implantes, como son la alteración del ACD $>10^{\circ}$ y el DTC $>5$ $\mathrm{mm}$, lo que causa una pérdida de la reducción en $16.9 \%$ de los casos fijados con placa, así como una mayor frecuencia de complicaciones postquirúrgicas tardías. 5,6,7 Sin embargo, estos parámetros son ambiguos respecto a la fijación con clavo centromedular. ${ }^{1,8}$ Por esta razón, con este estudio lo que se busca determinar son aquellas alteraciones en valores de medidas radiográficas estándar, como un factor que se asocia con la severidad de la lesión y con el porcentaje de complicaciones posteriores a la fijación con clavo centromedular. Asimismo, el objetivo del presente estudio es describir la frecuencia y magnitud de cambios radiográficos, al igual que la frecuencia de complicaciones en la osteosíntesis con clavo centromedular.

\section{Material y métodos}

Estudio de serie de casos, el cual se realizó en pacientes mayores de 18 años con fractura de húmero proximal de II, III y IV fragmentos de acuerdo con la clasificación de Neer. Los pacientes incluidos fueron tratados quirúrgicamente con osteosíntesis con clavo Targón PH (Aesculap, Tuttlingen, Alemania) durante el período de Enero de 2014 a Diciembre de 2017. Para ello, se realizaron valoraciones radiográficas seriadas en seguimiento postquirúrgico y se realizaron mediciones estándar (ACD, OM, DTC y ES) al inicio y al vicious consolidations in varus or valgus and increased presence of AVN.

Keywords: Proximal humeral fracture, treatment, nail, complications, X-rays. final del seguimiento radiográfico. Se excluyeron aquellos pacientes con un seguimiento menor a cuatro meses. Los pacientes fueron intervenidos en igual proporción por ocho cirujanos adscritos a nuestra institución.

Se analizaron por cada grupo características como edad, sexo, lateralidad de la lesión, tiempo de cirugía, tiempo de seguimiento y presencia de complicaciones. Se calcularon medidas tendencia central y dispersión (porcentajes, medias y desviaciones estándar) para comparar medidas escalares de muestras relacionadas; para ello, se utilizó la prueba de t de Student. Para variables nominales, se utilizó $\chi^{2}$. Los análisis se realizaron a través del programa estadístico SPSS versión 20 (IBM statistics).

El número de registro hospitalario por el Comité de Investigación es: CI/137/2019 30/19.

\section{Resultados}

De los 84 casos analizados, la relación entre sexo (femenino:masculino) fue de 3.2:1. No hubo diferencias respecto a la lateralidad. El seguimiento fue de 13.9 meses $( \pm 1.0)$ y la edad promedio de presentación de $65 \pm 2$ años. Existió una mayor frecuencia de casos en aquéllos con 70 a 79 años (34.5\%). El grado de severidad de la fractura (de acuerdo con la clasificación de Neer) presentó diferencias, donde $40.4 \%$ correspondió a grado II, en tanto que $29.7 \%$ (n $=25$ ) fue grado III o IV (Tabla 1).

La media inicial del ángulo cervicodiafisario (ACD) que se presentó fue de $139.5 \pm 1.4^{\circ}$. De forma general, $7.1 \%$ (n =6) presentó valgo $<120^{\circ}$ inicial, el cual aumentó hasta $10.7 \%(n=9)$ al final del seguimiento, aunque, en el análisis de casos, un grado III/IV de la clasificación de Neer existió hasta $46 \%$ en una angulación $<135^{\circ}$. La deformidad en valgo $>140^{\circ}$ se presentó en mayor frecuencia en pacientes Neer grado II con $44.1 \%(n=15 / 34)$ al inicio del seguimiento. Se observó que $30.9 \%$ tiene cambio de $\mathrm{ACD}(\triangle \mathrm{ACD}) \geq 10^{\circ}$, siendo más frecuente $(\mathrm{n}=19 / 50$ o $38 \%)$ en pacientes con lesiones graves (Neer grado III/IV), a diferencia de Neer grado II $(\mathrm{n}=7 / 34$ o $20.6 \%)(\mathrm{p}=0.043)($ Figura 1$)$.

\section{Offset medial}

Los valores normales que se consideran en la literatura ${ }^{9}$ (de 4 a $14 \mathrm{~mm})$ se identificaron en 42.9\% $(\mathrm{n}=36 / 84)$ al inicio 
del seguimiento. Una alta proporción ( $\mathrm{n}=48 / 84$ o $57.1 \%)$ se encontró con mediciones por debajo de lo normal $(<4 \mathrm{~mm})$, la cual se acentuó en Neer grado II $(n=24 / 34$ o $70.6 \%)$. En $94 \%$ de los casos $(n=79)$, no se observaron cambios mayores de $5 \mathrm{~mm}$ respecto a la valoración inicial y final.

\section{Distancia tuberosidad-cabeza}

La medición de la DTC inicial $<6 \mathrm{~mm}$ se reportó en $56.0 \%(n=47 / 84)$; sin embargo, los valores anatómicos $(6$ a $10 \mathrm{~mm}$ ) se reportaron únicamente en $26.2 \%$ de los casos. Los valores DTC $>10 \mathrm{~mm}$ al final del seguimiento son frecuentes en Neer grado III/IV (14\%); generalmente, se asocian con una consolidación viciosa proximal del troquiter, lo que causa un ascenso de éste. En $86.9 \%$ se tienen variaciones de DTC $(\triangle \mathrm{DTC})<5 \mathrm{~mm}$.

\section{Espacio subacromial}

El promedio de espacio subacromial (ES) inicial fue de $10.8 \pm 0.5 \mathrm{~mm}$, con un $\Delta$ ES global de $1.0 \pm 0.7 \mathrm{~mm}$. Sin embargo, se encontró ES $<10 \mathrm{~mm}$ inicial hasta en $41.7 \%$. Por clasificación de Neer grado II, se tuvo una media de $\Delta E S$ $2.1 \pm 0.48 \mathrm{~mm}$, en tanto que el grupo de Neer grado III/IV tuvo una media de $\Delta \mathrm{ES} 4.99 \pm 0.89 \mathrm{~mm}(\mathrm{p}=0.03)$.

Las complicaciones más frecuentes fueron la necrosis avascular (NAV) con $44 \%$, seguido de la atrofia muscular (14.3\%), seudoartrosis $(4.8 \%)$ y aflojamiento (3.6\%). Cuatro pacientes $(4.8 \%)$ requirieron reintervención con retiro de material por dolor (Figura 2).

En cuanto a la NAV, la presentación más frecuente dentro del estudio fue grado 2 de acuerdo con la clasificación de Cruess con 21 casos, mientras que 16 casos se presentaron con necrosis avanzada (Cruess 3 y 4). En cuanto a la severi- dad de la fractura, $58 \%$ de los casos de necrosis se presentó en Neer grado III/IV, en tanto que únicamente se obtuvieron ocho casos de necrosis en pacientes con Neer grado II.

\section{Discusión}

El presente estudio reporta una serie de casos en donde se manejaron fracturas de húmero proximal con clavo centromedular. De manera global, se ha observado un incremento en la frecuencia de presentación de fracturas de húmero proximal y hasta $250 \%$ más en países desarrollados, pues esto se ha relacionado con el aumento de la expectativa de vida. ${ }^{10} \mathrm{El}$ manejo quirúrgico aún sigue siendo controversial. ${ }^{11,12,13}$

La distribución de las características demográficas de nuestra serie es comparable con lo reportado en la literatura, pues, por un lado, hay una mayor frecuencia de casos que se da en mujeres y en pacientes de la tercer edad, sin diferencia de la lateralidad..$^{14}$ Por otro lado, no presentamos diferencias en proporción de casos por clasificación de Neer; si bien hay algunos reportes donde predominan los casos de baja severidad (Neer grado II), ${ }^{11,15,16,17,18,19}$ generando un posible sesgo de resultados. Kloub y colaboradores, ${ }^{20}$ así como Mathews y otros ${ }^{21}$ presentaron dos cohortes de seguimiento $(n=137$ cada una) y Targon ${ }^{\circledR} \mathrm{PH}$ (Aesculap, Tuttlingen, Alemania) utilizado en pacientes con Neer grado III/IV. Lo que ellos reportaron fue una reducción no anatómica en $7.3 \%$, pérdida de la reducción en $2.9 \%$ y reintervención en $4.4 \%$ de los casos. Por su parte, Wong y su equipo de investigación, con una cohorte más grande $(n=385)$, reportaron el uso de distintas marcas de clavo centromedular y frecuencias más altas en reintervención (15.8\%), además de pérdidas de la reducción $(9.9 \%)$, lo que evidencia la variabilidad de resultados al utilizar este tipo de implantes.

\begin{tabular}{|c|c|c|c|c|c|c|c|c|c|}
\hline & & \multicolumn{8}{|c|}{ Clasificación de Neer } \\
\hline & & \multicolumn{2}{|c|}{$\begin{array}{c}\text { II } \\
(\mathrm{N}=34)\end{array}$} & \multicolumn{2}{|c|}{$\begin{array}{c}\text { III } \\
(\mathrm{N}=25)\end{array}$} & \multicolumn{2}{|c|}{$\begin{array}{c}\text { IV } \\
(\mathrm{N}=25)\end{array}$} & \multicolumn{2}{|c|}{$\begin{array}{c}\text { Total } \\
(\mathrm{N}=84)\end{array}$} \\
\hline & & $\mathrm{n}$ & $\%$ & $\mathrm{n}$ & $\%$ & $\mathrm{n}$ & $\%$ & $\mathrm{n}$ & $\%$ \\
\hline \multirow{2}{*}{ Género } & Femenino & 25 & 73.5 & 21 & 84.0 & 18 & 72.0 & 64 & 76.2 \\
\hline & Masculino & 9 & 26.5 & 4 & 16.0 & 7 & 28.0 & 20 & 23.8 \\
\hline \multirow[t]{2}{*}{ Lateralidad } & Derecho & 17 & 50.0 & 10 & 40.0 & 14 & 56.0 & 41 & 48.8 \\
\hline & Izquierdo & 17 & 50.0 & 15 & 60.0 & 11 & 44.0 & 43 & 51.2 \\
\hline \multirow[t]{8}{*}{ Grupo de edad (años) } & $18-29$ & 1 & 2.9 & 0 & 0.0 & 3 & 12.0 & 4 & 4.8 \\
\hline & $30-39$ & 5 & 14.7 & 0 & 0.0 & 3 & 12.0 & 8 & 9.5 \\
\hline & $40-49$ & 0 & 0.0 & 0 & 0.0 & 4 & 16.0 & 4 & 4.8 \\
\hline & $50-59$ & 0 & 0.0 & 5 & 20.0 & 2 & 8.0 & 7 & 8.3 \\
\hline & $60-69$ & 8 & 23.5 & 3 & 12.0 & 5 & 20.0 & 16 & 19.0 \\
\hline & $70-79$ & 12 & 35.3 & 12 & 48.0 & 5 & 20.0 & 29 & 34.5 \\
\hline & $80-89$ & 3 & 8.8 & 4 & 16.0 & 3 & 12.0 & 10 & 11.9 \\
\hline & $>90$ & 5 & 14.7 & 1 & 4.0 & 0 & 0.0 & 6 & 7.1 \\
\hline Seguimiento (meses) & Media (DE) & $14.0(1.8)$ & & $11.9(1.5)$ & & $15.8(2.0)$ & & $13.9(1.0)$ & \\
\hline
\end{tabular}




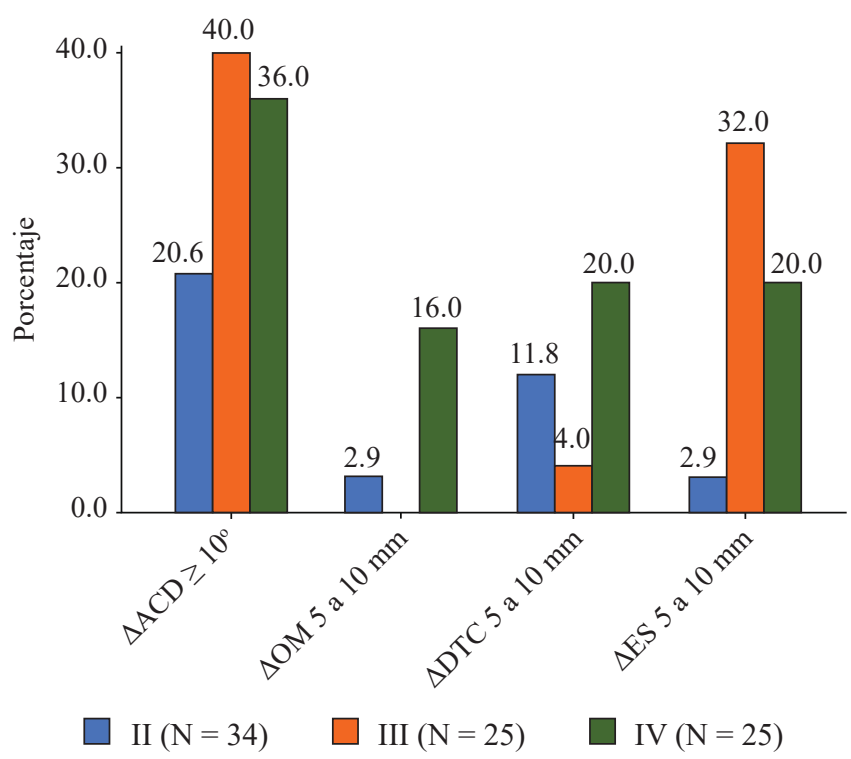

Figura 1: Frecuencia de cambios en mediciones radiográficas de acuerdo con la clasificación de Neer.

Las medias de mediciones radiográficas $(\mathrm{ACD}, \mathrm{ES}$, DTC y OM) de este estudio se encuentran dentro de los promedios, los cuales han sido identificados para distintos implantes por los autores ya mencionados $1,8,11,15,16,19,22$ (Tabla 2). La alteración del ACD ha sido asociado con la rotación interna del húmero durante las proyecciones radiográficas como el factor que afecta dichos valores. , 23,24 $^{-1}$ Otros estudios anatómicos hechos en cadáver reportaron que en radiografías simples no se afecta la medición de ACD con la posición del brazo. ${ }^{25}$ Por lo anterior, los estudios clínicos han determinado el punto de corte de $\triangle \mathrm{ACD}$ $>10^{\circ}$, así como el $\Delta \mathrm{DTC}>5 \mathrm{~mm}$, para tener un impacto clínico y un predictor de pérdida de la fijación. ${ }^{4}$ En nuestro estudio, los pacientes que presentaron alteraciones $\mathrm{ACD}>$ $10^{\circ}$ con mayor frecuencia fueron los Neer grado III y IV, con hasta 40 y $36 \%$ de los casos durante el seguimiento. Esto es similar a lo reportado por Ueyama y colaboradores $^{17}$ y por Tsitsilonis y otros, ${ }^{22}$ donde los cambios en ACD se asociaron tanto con la severidad de fractura como con el sitio de entrada lateral del clavo.

Respecto a la deformidad en varo $\left(<135^{\circ}\right)$, encontramos hasta $46 \%$ de los casos Neer grado III y IV, muy similar a los estudios de Nolan y su grupo ${ }^{16}$ y Tsitsilonis y otros, ${ }^{22}$ donde se asoció la severidad de la fractura con ángulos postquirúrgicos de hasta $117.9^{\circ}$; casos menores de $120^{\circ}$ en este estudio fueron poco frecuentes $(6 \%)$.

La medición de OM, ES y DTC tuvieron modificaciones en casos Neer grado IV. Al respecto, Ueyama y colaboradores ${ }^{17}$ lo asocian con una alta proporción de pacientes grado IV de la clasificación de Neer, contrario a Thomazeau, ${ }^{8}$ quien lo relaciona con una mala selección de los pacientes, e incluso recomienda no utilizar este tipo de implante en esta clasificación de pacientes (Neer IV).
El presente estudio reporta una alta frecuencia de complicaciones a largo plazo (necrosis avascular, seudoartrosis y aflojamiento). Asimismo, pocos pacientes presentaron complicaciones en el período postquirúrgico temprano (infección de sitio quirúrgico o lesión neurológica) (Figura 2). En contraste con la literatura, las series de pacientes más grandes que usan clavo centromedular reportaron tasas de complicaciones de 26 a 59\%. Con lo anterior, se identifica que el tipo de fractura es propenso a una alta tasa de complicaciones relacionadas, tanto con la severidad de la lesión como con el riesgo inherente de la personalidad de la fractura. ${ }^{1,20,21,26}$

La complicación más frecuente que reportamos fue la NAV, principalmente en Neer grado III/IV y con edad avanzada. A pesar de existir amplios estudios que indican los factores de riesgos vascular, dentro de los cuales se indica la severidad de la fractura, ${ }^{27}$ Beck y colaboradores indican que existe una asociación de la NAV con la manipulación por el procedimiento quirúrgico, independiente del grado de severidad de la fractura, ya que altera la vascularidad residual por la manipulación. ${ }^{12}$ En cuanto a otras complicaciones, como la consolidación viciosa, aflojamiento y pérdida de la reducción, nuestros resultados se encuentran dentro de parámetros similares a la serie de Wong. ${ }^{28}$ Nuestra tasa de reintervención quirúrgica fue similar a Mathews ${ }^{21}(4.4 \%)$, con una menor frecuencia a la reportada por Gadea ${ }^{3}(25.4 \%)$, Gracitelli ${ }^{15}$ (18.8\%) o Wong $^{28}(15.8 \%)$.

Una de las limitantes de este estudio es la integración de escalas funcionales prequirúrgicas a los pacientes, lo que se requiere para estandarizar resultados con respecto a otros países. También, por tratarse de un estudio de concentración de casos, estimar valores nacionales es complicado.

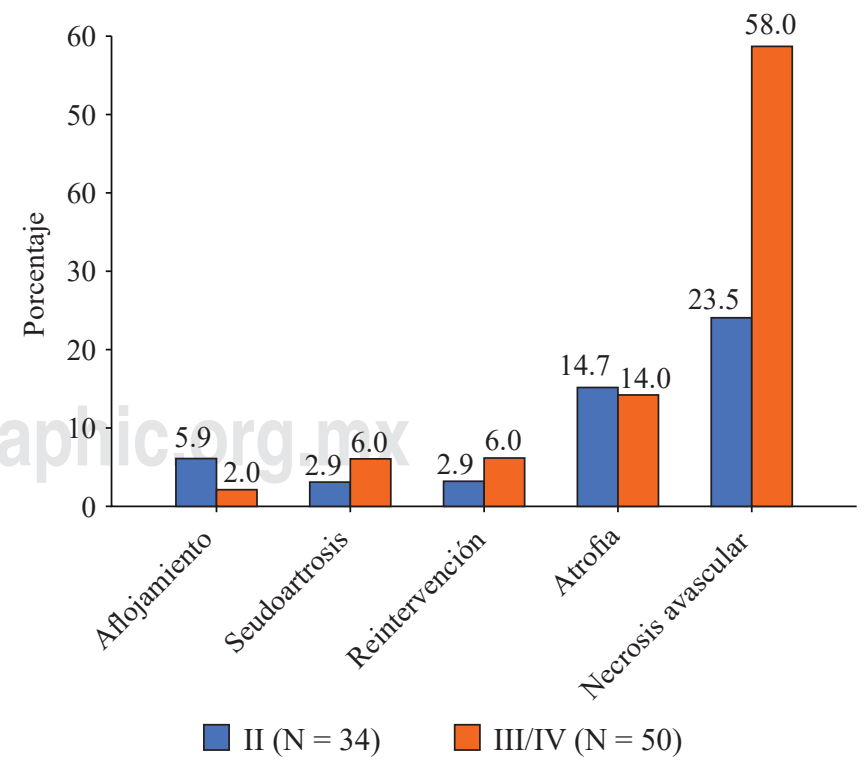

Figura 2: Frecuencia de presentación de complicaciones de acuerdo con la clasificación de Neer. 
Tabla 2: Resultados de las mediciones radiográficas de acuerdo con clasificación de Neer y el tipo de clavo centromedular utilizado.

\begin{tabular}{|c|c|c|c|c|c|}
\hline Estudio & Tipo de clavo & $\mathrm{n}$ & Clasificación de Neer & \multicolumn{2}{|c|}{ Resultados de mediciones } \\
\hline Gracitelli, et al. ${ }^{15}$ & $\begin{array}{l}\text { Centronail }^{\circledR} \text { (Orthofix, } \\
\text { Verona, Italia) }\end{array}$ & 32 & II y III & $\mathrm{ACD}$ & $\begin{array}{l}\left.133.7^{\circ} \pm 7.3^{\circ} \text { (inmediato }\right) \\
133.7^{\circ} \pm 7.3^{\circ}(12 \text { meses })\end{array}$ \\
\hline \multirow{3}{*}{ Nolan, et al. ${ }^{16}$} & \multirow{3}{*}{$\begin{array}{l}\text { Polaris nail } 1^{\mathbb{}} \text { (Acumed, } \\
\text { Beaverton, OR) }\end{array}$} & \multirow{3}{*}{18} & II & $\mathrm{ACD}$ & $\begin{array}{l}132.5^{\circ}(120-150) \text { Inicial } \\
124.4^{\circ}(90-150) \text { final }\end{array}$ \\
\hline & & & \multirow[t]{2}{*}{ III } & & $\begin{array}{l}127.6^{\circ}(125-130) \text { inicial } \\
113.8^{\circ}(100-130) \text { final }\end{array}$ \\
\hline & & & & DTC & $\begin{array}{l}50 \% \text { alteración de DCT } 5-10 \mathrm{~mm} \\
50 \% \mathrm{ACD}<120^{\circ} \text { al final del seguimiento }\end{array}$ \\
\hline Tsitsilonis, et al. ${ }^{22}$ & $\begin{array}{l}\text { PHN system }\left(\text { Targon }^{\circledR}\right. \\
\text { Aesculap) }\end{array}$ & 43 & II, III y IV & $\mathrm{ACD}$ & $\begin{array}{l}\text { Total }(n=43) 126.5^{\circ} \pm 16.9^{\circ} \\
\text { Neer grado II }(n=10) 130.8^{\circ} \pm 21.2^{\circ} \\
\text { Neer grado III }(n=23) 128.4^{\circ} \pm 14.3^{\circ} \\
\text { Neer grado IV }(n=10) 117.9^{\circ} \pm 16.7^{\circ}\end{array}$ \\
\hline Ueyama, et al. ${ }^{17}$ & Aesculap Targon ${ }^{\circledR}$ PH-P & 15 & II y III & $\mathrm{ACD}$ & $\begin{array}{l}\triangle \mathrm{ACD} \text { promedio } 4.6^{\circ}(\mathrm{DE} 11.0 ; 0-45) \\
\text { Sitio de entrada lateral } \triangle \mathrm{ACD} 13.8^{\circ}(0-45) \\
6.7 \% \text { desplazamiento }\left(>15^{\circ} \text { en } \mathrm{ACD}\right)\end{array}$ \\
\hline Hatzidakis, et al. ${ }^{24}$ & 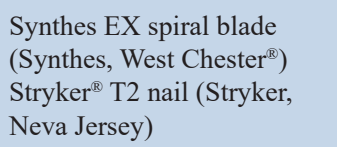 & 38 & II & $\mathrm{ACD}$ & $\begin{array}{l}\text { Promedio } 131^{\circ}(126-132) \\
\triangle \mathrm{ACD} 3.2^{\circ} \\
133.6 \pm 4.3 \rightarrow 130.4 \pm 8.9 ; \mathrm{p}=0.04 \\
\text { Reoperación } 13 \%(3-24 \%)\end{array}$ \\
\hline Park, et al. ${ }^{19}$ & $\begin{array}{l}\text { Uniflex }^{\circledR} \text { (Biomet, IN, EUA) } \\
\text { Polarus nail }\end{array}$ & 43 & III & $\mathrm{ACD}$ & $\begin{array}{l}\text { Postquirúrgico inicial } 141^{\circ} \pm 9.9^{\circ} \\
\text { Postquirúrgico final } 137 \pm 11.1\end{array}$ \\
\hline Trepat, et al. ${ }^{11}$ & $\begin{array}{l}\text { T2 Proximal Humeral Nail } \\
\text { (Stryker }^{\text {B }} \text {, Neva Jersey) }\end{array}$ & 15 & II & $\mathrm{ACD}$ & $\begin{array}{l}\text { Inmediato } 123^{\circ}(90-140) \\
\text { A un año, } 121^{\circ}(90-130) \\
\text { Dos pacientes en varo }\left(<110^{\circ}\right) \text { por falla de } \\
\text { la reducción }\end{array}$ \\
\hline
\end{tabular}

\section{Conclusiones}

1. Las mediciones radiográficas presentan cambios en valoraciones seriadas a largo plazo. La medida radiográfica con mayor alteración es el ACD, cuya tendencia es la de consolidación en varo $\left(<120^{\circ}\right)$ al final del seguimiento.

2. Las alteraciones en $\operatorname{ACD}\left(>10^{\circ}\right)$ y $\operatorname{ES}(>5 \mathrm{~mm})$ se dan en mayor proporción en aquellos pacientes Neer grado III/ IV; esto va acorde con la severidad de la fractura.

3. A pesar de que la modificación de DTC, OM y ES se presenta en baja frecuencia, el ES estrecho $(<10 \mathrm{~mm})$ sí tiene alta prevalencia y representa un factor de riesgo para pinzamiento subacromial. ${ }^{29}$

4. Con excepción de la NAV, el clavo centromedular en este estudio presenta baja proporción de complicaciones, lo cual es compatible con lo reportado por la literatura.

\section{Bibliografía}

1. Wong J, Newman JM, Gruson KI. Outcomes of intramedullary nailing for acute proximal humerus fractures: a systematic review. J Orthop Traumatol. 2016; 17: 113-22.
2. Canbora MK, Kose O, Polat A, et al. Relationship between the functional outcomes and radiological results of conservatively treated displaced proximal humerus fractures in the elderly: A prospective study. Int J Shoulder Surg. 2013; 7: 105-9.

3. Gadea F, Favard L, Boileau P, et al. Fixation of 4-part fractures of the proximal humerus: Can we identify radiological criteria that support locking plates or IM nailing? Comparative, retrospective study of 107 cases. Orthop Traumatol Surg Res. 2016; 102: 963-70.

4. Bai L, Fu ZG, Wang TB, et al. Radiological evaluation of reduction loss in unstable proximal humeral fractures treated with locking plates. Orthop Traumatol Surg Res. 2014; 100: 271-4.

5. Jung SW, Shim S-B, Kim HM, et al. Factors that influence reduction loss in proximal humerus fracture surgery. J Orthop Trauma. 2015; 29: $276-82$

6. Jia XY, Chen YX, Qiang M-F, et al. Postoperative evaluation of reduction loss in proximal humeral fractures: a comparison of plain radiographs and computed tomography. Orthop Surg. 2017; 9: 167-73.

7. Newton AW, Selvaratnam V, Pydah SK, et al. Simple radiographic assessment of bone quality is associated with loss of surgical fixation in patients with proximal humeral fractures. Injury. 2016; 47: 904908.

8. Thomazeau H, Richou J, Benkalfalte T, et al. Is it worth fixing proximal humeral fractures at increased vascular risk? Orthop Traumatol Surg Res. 2012; 98: 383-9.

9. Pearl ML, Volk AG. Coronal plane geometry of the proximal humerus relevant to prosthetic arthroplasty. J Shoulder Elbow Surg. 1996; 5: $320-6$. 
10. Patel S, Colaco HB, Elvey ME, et al. Post-traumatic osteonecrosis of the proximal humerus. Injury. 2015; 46: 1878-84.

11. Trepat AD, Popescu D, Fernández-Valencia JA, et al. Comparative study between locking plates versus proximal humeral nail for the treatment of 2-part proximal humeral fractures. Eur J Orthop Surg Traumatol. 2012; 22: 373-9.

12. Beks RB, Ochen Y, Frima H, et al. Operative versus nonoperative treatment of proximal humeral fractures: a systematic review, metaanalysis, and comparison of observational studies and randomized controlled trials. J Shoulder Elbow Surg. 2018; 27: 1526-34.

13. Sun Q, Ge W, Li G, et al. Locking plates versus intramedullary nails in the management of displaced proximal humeral fractures: a systematic review and meta-analysis. Int Orthop. 2018; 42: 641-50.

14. Passaretti D, Candela V, Sessa P, et al. Epidemiology of proximal humeral fractures: a detailed survey of 711 patients in a metropolitan area. J Shoulder Elbow Surg. 2017; 26(12): 2117-24. doi: 10.1016/j. jse.2017.05.029.

15. Gracitelli MEC, Malavolta EA, Assunção JH, et al. Locking intramedullary nails compared with locking plates for two- and three-part proximal humeral surgical neck fractures: a randomized controlled trial. J Shoulder Elbow Surg. 2016; 25: 695-703.

16. Nolan BM, Kippe MA, Wiater JM, et al. Surgical treatment of displaced proximal humerus fractures with a short intramedullary nail. J Shoulder Elbow Surg. 2011; 20: 1241-7.

17. Ueyama H, Yano K, Kanemura M, et al. Clinical outcomes and prognostic factors depending on implant design in the treatment of proximal humeral fractures: a retrospective study. J Orthop. 2016; 13: 369-75.

18. Dilisio MF, Nowinski RJ, Hatzidakis AM, et al. Intramedullary nailing of the proximal humerus: evolution, technique, and results. J Shoulder Elbow Surg. 2016; 25: e130-8.

19. Park J-Y, Kim J-H, Lhee S-H, et al. The importance of inferomedial support in the hot air balloon technique for treatment of 3-part proximal humeral fractures. J Shoulder Elbow Surg. 2012; 21: 1152-9.

20. Kloub M, Holub K, Polakova S. Nailing of three- and four-part fractures of the humeral head-Long-term results. Injury. 2014; 45: S29-S37.
21. Mathews J, Lobenhoffer P. Osteosynthese instabiler proximaler Humerusfrakturen mit dem Targon ${ }^{\mathbb{B}}-\mathrm{PH}-\mathrm{Nagel}$. Oper Orthop Traumatol. 2007; 19: 255-75.

22. Tsitsilonis S, Schaser K-D, Kiefer H, et al. The treatment of the proximal humeral fracture with the use of the PHN Nailing System: the importance of reduction. Acta Chir Orthop Traumatol Cech. 2013; 80: 250-5.

23. Assunção JH, Malavolta EA, Beraldo RA, et al. Impact of shoulder rotation on neck-shaft angle: A clinical study. Orthop Traumatol Surg Res. 2017; 103: 865-8.

24. Hatzidakis AM, Shevlin MJ, Fenton DL, et al. Angular-stable locked intramedullary nailing of two-part surgical neck fractures of the proximal part of the humerus: a multicenter retrospective observational study. J Bone Jt Surg-Am. 2011; 93: 2172-9.

25. Malavolta EA, Assunção JH, Pagotto RA, et al. The rotation of the humeral head does not alter radiographic evaluation of the head-shaft angle. J Shoulder Elbow Surg. 2016; 25: 543-7.

26. Blum J, Hansen M, Müller M, et al. Proximal humeral fractures and intramedullary nailing: Experience with a new nail system. Eur $J$ Trauma Emerg Surg. 2009; 35: 489-98.

27. Campochiaro G, Rebuzzi M, Baudi P, et al. Complex proximal humerus fractures: Hertel's criteria reliability to predict head necrosis. Musculoskelet Surg. 2015; 99: 9-15.

28. Wong J, Newman JM, Gruson KI. Outcomes of intramedullary nailing for acute proximal humerus fractures: a systematic review. J Orthop Traumatol Off J Ital Soc Orthop Traumatol. 2016; 17: 113-22.

29. Calori GM, Colombo M, Bucci MS, et al. Complications in proximal humeral fractures. Injury. 2016; 47: S54-8.

Conflicto de intereses: En el presente estudio, no existe conflicto de intereses de ninguno de los autores para su realización. 